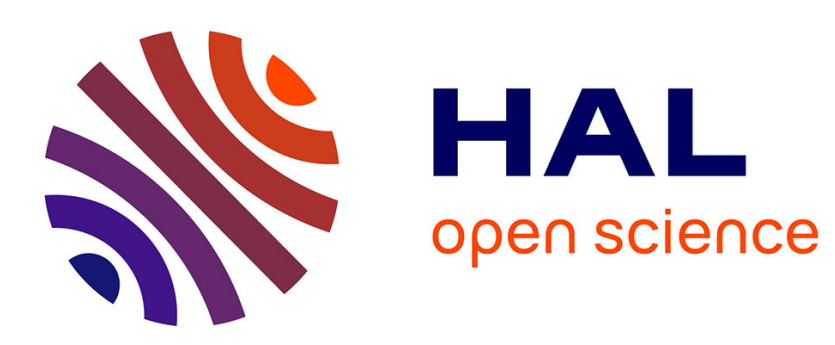

\title{
Transport of Long Neutral Polymers in the Semidilute Regime through a Protein Nanopore
}

Abdel Ghani Oukhaled, Anne-Laure Biance, Juan Pelta, Loïc Auvray, Laurent Bacri

\section{- To cite this version:}

Abdel Ghani Oukhaled, Anne-Laure Biance, Juan Pelta, Loïc Auvray, Laurent Bacri. Transport of Long Neutral Polymers in the Semidilute Regime through a Protein Nanopore. Physical Review Letters, 2012, 108 (8), pp.088104. 10.1103/PhysRevLett.108.088104 . hal-02006520

\author{
HAL Id: hal-02006520 \\ https://hal.science/hal-02006520
}

Submitted on 22 Mar 2019

HAL is a multi-disciplinary open access archive for the deposit and dissemination of scientific research documents, whether they are published or not. The documents may come from teaching and research institutions in France or abroad, or from public or private research centers.
L'archive ouverte pluridisciplinaire HAL, est destinée au dépôt et à la diffusion de documents scientifiques de niveau recherche, publiés ou non, émanant des établissements d'enseignement et de recherche français ou étrangers, des laboratoires publics ou privés. 


\title{
Transport of long neutral polymers in the semi-dilute regime through a protein nanopore
}

\author{
Abdel Ghani Oukhaled, ${ }^{1}$ Anne-Laure Biance, ${ }^{2}$ Juan Pelta, ${ }^{1}$ Loic Auvray,${ }^{3}$ and Laurent Bacri ${ }^{1, *}$ \\ ${ }^{1}$ LAMBE UMR 8587 CNRS, Évry and Cergy University, France \\ ${ }^{2}$ LPMCN UMR 5586 CNRS, Université de Lyon, France \\ ${ }^{3}$ MSC UMR 7057 CNRS, Paris Diderot University, France
}

(Dated: November 21, 2011)

\begin{abstract}
We investigate the entrance of single poly(ethylene glycol) (PEG) chains into an $\alpha$-Hemolysin channel. We detect the frequency and duration of the current blockades induced by large neutral polymers, where chain radius is larger than pore diameter. In the semi-dilute regime, these chains pass only if the monomer concentration is larger than a well-defined threshold. Experiments are performed in a very large domain of concentration and molecular mass, up to $35 \%$ and $200 \mathrm{kDa}$ respectively, previously unexplored. The variation of the dwell time as a function of molecular mass shows that the chains are extracted from the semi-dilute solution in contact with the pore by a reptation mechanism.
\end{abstract}

PACS numbers: 82.35.Pq, 87.16.dp, 36.20.Ey

All experiments concerning the dynamics of macromolecules, neutral polymers [1-12], polyelectrolytes [1317], single strand DNA [18, 19] or RNA[20], unfolded proteins [21-23] through protein nanopores have been performed in dilute solution using the patch-clamp technique [24]. Cell nucleus, cytoplasm and extra-cellular matrix are semi-dilute media, where biomolecules are entangled. Theoretical predictions describe the dynamics of neutral polymers mainly in the dilute solution [2529], but rarely in the semi-dilute regime [30]. In this regime and when the radius of the chain is larger than the pore diameter, the molecules are expected to enter the pore only if the polymer concentration is sufficiently large [31]. The osmotic pressure due to the polymer network must overcome the force necessary to confine one chain in the nanopore. This behavior has already been observed experimentally in porous media by neutron scattering [32-34]. The dwell time is also expected to be different in this semi-dilute regime and governed by the extraction of one single chain of the polymer network into the nanopore. Consequently, it is expected that the dwell time will increase with the chain monomer number $N$ as $\tau=\tau_{0} c^{1.5} N^{3}$, according to a reptation model [25].

Previous experiments on the transport of neutral polymers (PEG) have always been performed in a range of low molecular masses $(\mathcal{M}<10 k D a)$ and relatively low mass concentration $(c \leq 15 \%)$, in a dilute regime where the chains are not entangled. The pioneering experiment on molecular counting was performed by Bezrukov [4] and the first detection of RNA and DNA by Kasianowicz [20]. More recently, Bezrukov has carried out experiments concerning the transport of PEG in a dilute regime. If the polymers are too large to be accommodated within the pore, the out-of-the-pore part of the molecule pulls on the trapped part, thus acting as an entropic spring [35].

In this work, we explore experimentally the transport of very long polymers in semi-dilute regime through an

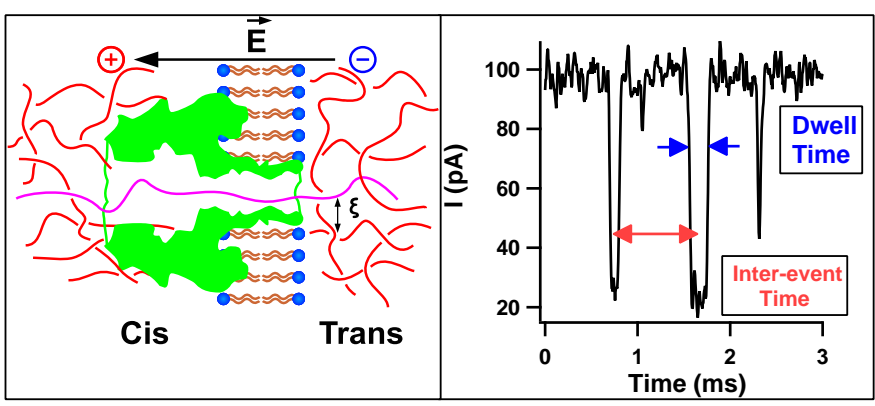

FIG. 1. (left) $\alpha$-Hemolysin channel inserted into lipid bilayers in presence of long polymers in semi-dilute regime. $\xi$ is the mesh size of this solution. (right) Single-channel recordings of the pore in the presence of PEG $2 \mathrm{kDa} 20 \%$ (w/v) applied to both sides of the bilayer. The applied voltage is $100 \mathrm{mV}$.

$\alpha$-Hemolysin nanopore (Fig. 1). Lipid bilayers are prepared using a classical method [36]. We use a $1 \mathrm{M} \mathrm{KCl}$, $5 \mathrm{mM}$ HEPES pH 7.4 buffer. The ionic current is detected using an Axopatch 200B amplifier by applying a constant voltage of $100 \mathrm{mV}$. The acquisition frequency is $100 \mathrm{kHz}$. The data are filtered at $10 \mathrm{kHz}$ and processed by dedicated software (Igor Pro, Wavemetrics). The first experiments are conducted with PEG of low molecular masses $(\mathcal{M} \leq 10 \mathrm{kDa})$ in dilute solution (table 1 supplementary material). The PEG chains are added to both compartments in equal amounts. We increase the mass of the molecules to study the dynamics of their entry into the pore. We check with short chains $(2 \mathrm{kDa})$ that the event frequency varies linearly with polymer concentration, in agreement with previous observations [10]. The dwell time (defined in the Fig. 1) does not vary as a function of the polymer concentration (supplementary data). To determine the influence of the molecular mass on the event frequency, we compare the recorded current traces of $25 \mathrm{mM}$ PEG 1, 2 and $3.4 \mathrm{kDa}$. A decrease in the event 


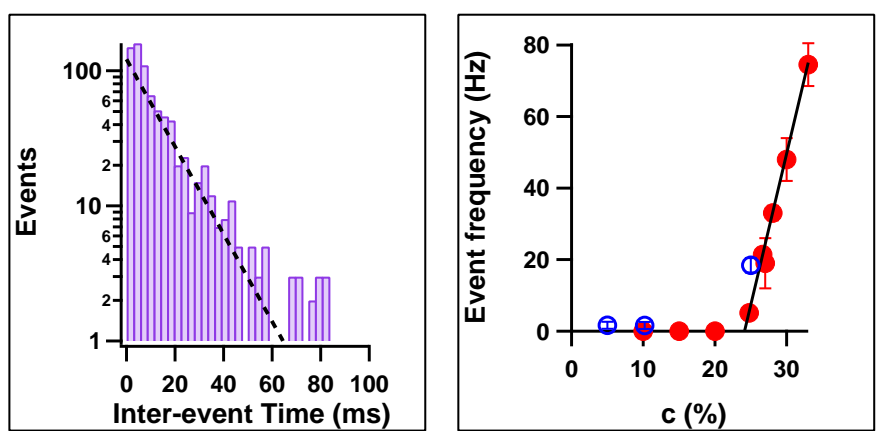

FIG. 2. Event frequency of long neutral polymers. (left) Histogram of the inter-event time of a PEG $35 \mathrm{kDa}$ solution $(33 \%)$. The event frequency $(75 \pm 6 \mathrm{~Hz})$ is estimated from the exponential fit (dotted line). (Right) Event frequency for the PEG $20 \mathrm{kDa}$ (blue diamonds) and $35 \mathrm{kDa}$ (red circles) according to their mass concentration.

frequency is observed when the chain size increases. As expected theoretically $[26,37]$ due to the free energy required for a chain to enter a nanopore and as already observed experimentally $[8,10]$, we check that the logarithm of the equilibrium association constant $\log \left(K_{f}\right)$ is inversely proportional to the molecular mass of the polymer (supplementary data). Moreover, large molecular mass $(\geq 10 \mathrm{kDa})$ polymer transport is not observed in the range of weak concentrations (lower than $15 \%$ ), as described in previous experiments [8].

In order to force the entry of long chains, it is necessary to increase the PEG concentration, which becomes greater than the overlap concentration $c^{*}$, calculated by the relationship $c^{*}=N /\left[(4 / 3) \pi R_{g}^{3}\right][25]$ where $R_{g}$ is the radius of gyration of the chain (supplementary material). From this, we deduce the overlap mass concentration $c^{*}(\mathrm{w} / \mathrm{v})=\mathcal{M} / \mathcal{N}_{A}\left[(4 / 3) \pi R_{g}^{3}\right]$, where $\mathcal{N}_{A}$ is Avogadros Number. As $c>c^{*}$, the polymer chains are in the semi-dilute regime, characterized by the mesh size $\xi$ of the polymer network [25] given by the scaling relation $\xi=a \phi^{-0.75} . a=0.35 \mathrm{~nm}$ is the size of each monomer, taking into account the interactions with the solvent. $\phi$ is the volume fraction of polymer given by $\phi=\rho_{w} / \rho c$, with $\rho$ the chain density, almost equal to the density of water $\rho_{w}: \xi=a c^{-0.75}$.

The pressure force pushing the chains into the pore is $F_{\Pi} \simeq \Pi D^{2}$ where $\Pi$ is the osmotic pressure and $D$ the pore diameter. This pressure force must overcome the confinement force of the chain in a channel of radius $D$ : $F_{\text {Conf }} \simeq \frac{k_{B} T}{D}$. The pressure $\Pi$ must therefore be bigger than $\frac{k_{B} T}{D^{3}}$. As $\Pi \simeq \frac{k_{B} T}{\xi^{3}}$ in the semi-dilute regime [25], we deduce that $\xi$ must be smaller than $D$ for chains to enter the pore.

In the case of $35 \mathrm{kDa}$ PEG, we vary the polymer concentration from $10 \%$ to $33 \%$ and we report event frequency versus polymer concentration (Fig. 2). No block- ade is observed up to $25 \%$ polymer concentration, (Fig. 2right). Above $25 \%$, the event frequency $f$ increases with concentration. From the intersection between this curve and the bottom axes, we determine a threshold concentration $c_{t h}=24 \pm 2.6 \%$ by linear extrapolation.

The estimated radius of gyration of $35 \mathrm{kDa}$ PEG in dilute solution is $7.9 \mathrm{~nm}$, which is very large compared to the $\alpha$-Hemolysin pore radius $(1 \mathrm{~nm})$. The polymer cannot enter the pore in the dilute regime ( $c$ lower than $\left.c^{*}=2.9 \%\right)$. When the concentration is increased above $c^{*}$, the polymer network mesh size (or correlation length) $\xi$ decreases. At the threshold concentration observed here, $c_{t h}=24 \pm 2.6 \%$, we calculate $\xi\left(c_{t h}\right)=1.05 \pm 0.1 \mathrm{~nm}$, a value very close to the radius of the $\alpha$-Hemolysin pore. This key result is in very good agreement with de Gennes' theory [25]. Experiments are also performed with $20 \mathrm{kDa}$ PEG (Fig. 2) and $10 \mathrm{kDa}$ PEG. Translocation events are observed if polymer concentration is above $25 \%$ and $15 \%$ respectively. In both cases, polymer entry into the channel is observed only if the correlation length $\xi$ is smaller than the pore radius. Note that the theory of Daoud and de Gennes [26] applies only for very long pores, much longer than the chain, which is clearly not the case here. However, a threshold is observed because the free energy of confined chains over the length of the pore is much larger than the thermal energy $k_{B} T$.

An important feature of polymer dynamics in the nanopore is the dwell time. This has been measured in the semi-dilute regime where the molecular mass varies between $10 \mathrm{kDa}$ and $200 \mathrm{kDa}$. Experiments are carried out with pure solutions when molecular masses are lower than $35 \mathrm{kDa}$; otherwise mixtures of $35 \mathrm{kDa}$ and 100 (or 200) $\mathrm{kDa}$ chains, with several chain concentrations, are studied. This procedure must be employed because of lipid bilayer fragility. We clearly observe two types of blockade: short ones lasting milliseconds and long events lasting hundreds of milliseconds (Fig. 4). We attribute the long ones to the $100 \mathrm{kDa}$ or $200 \mathrm{kDa}$, because they are not observed in the case of pure $35 \mathrm{kDa}$ solution.

This dwell time, reported in Fig. 3, increases with the molecular mass of the polymer. Two regimes are observed: the first one with molecular masses lower than $10 \mathrm{kDa}$ (Fig. 3). The dwell time increases slowly with $\mathcal{M}$ and is independent of molecular concentration. The diffusion dynamics are mainly governed by the dissipation inside the channel and not by the polymer behavior outside the pore. These dynamics are discussed in supplementary materials. In the second one, the dwell time of long chains $(\mathcal{M} \geq 10 k D a)$ increases faster with $\mathcal{M}$ and is moreover a function of molecular concentration.

To observe the dynamics of these large chains, all the experiments must be performed in the semi-dilute regime: the chains are entangled in a random network, the mesh size $\xi$ of which is smaller than the pore size. If the chain is long enough, the chain dynamics are governed by the reptation phenomenon [25]. In the reptation 

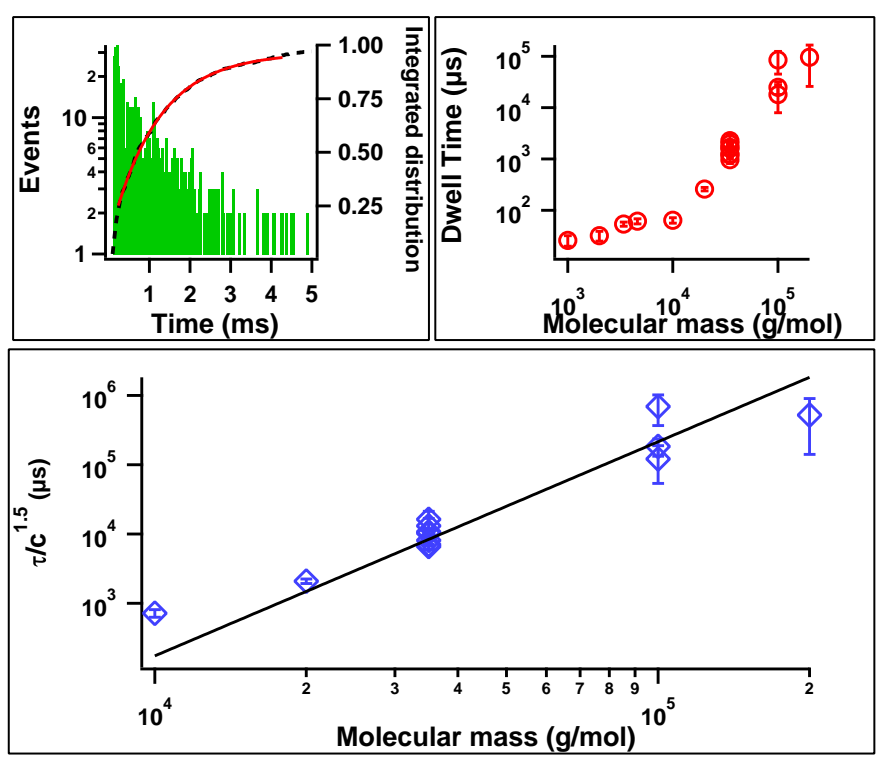

FIG. 3. Dynamics of neutral chains as a function of molecular mass (Left): Histogram of the dwell time of PEG $35 \mathrm{kDa}$ $(c=33 \%)$ and its integrated distribution. From the exponential fit (red curve), we estimate the dwell time: $\tau=$ $1250 \pm 100 \mu \mathrm{s}$. In the case of $100 \mathrm{kDa}$ and $200 \mathrm{kDa}$ PEG, we average $\tau$ with the mean dwell time. (Right): Dwell time $\tau$ versus PEG molecular mass $\mathcal{M} . c \leq 20 \%$ for $\mathcal{M}<10 k D a$ else $c>c_{t h}$. (Below): Reduced dwell time $\left(\frac{\tau}{c^{1.5}}\right)$ versus PEG molecular mass. The line corresponds to a power fit function $(\mathcal{M} \geq 35 k D a)$. The error bars are deduced from the exponential fits or from the dwell time distribution.

model, the tube length is $L=\frac{N}{N_{e}} \xi=\frac{N}{N_{e}^{0.5}} a$ where $N_{e}$ is the number of monomers in each blob. Firstly, we consider the $10 \mathrm{kDa}$ PEG at the threshold concentration. As $\xi=1.05 \pm 0.1 \mathrm{~nm}$, we calculate that $N_{e} \approx 9$ and $L \approx 27 \mathrm{~nm}$ : one third of the chain is confined inside the pore and the other two thirds is entangled in the semi-dilute solution. This case is situated at a crossover where both mechanisms take place together: dynamics inside the pore and outside the pore.

When the molecular mass is larger than $35 \mathrm{kDa}(N \geq$ $795)$, the tube length ( $L \geq 93 \mathrm{~nm}$ ) is longer than the pore length $10 \mathrm{~nm}$. Consequently, the dynamics of the chain in the $\alpha$-Hemolysin pore is dominated by the time necessary for the chain, engaged in the pore, to be extracted from the network of the other chains, and not by the confinement, which is neglected. The reptation time varies according to $N^{3}: \tau_{r e p}=\tau_{0} \frac{N^{3}}{N_{e}}$ where $\tau_{0}=\frac{6 \pi \eta a^{3}}{k_{B} T}=2 \times 10^{-4} \mu s$.

Note that the reptation time also varies versus the concentration through $N_{e}$, the number of monomers per blob. It reads: $\tau_{\text {rep }}=\frac{6 \pi \eta a^{3}}{k_{B} T} N^{3} \phi^{1.5}$. We then define a reduced time as $\tau_{r e p} / c^{1.5}=\left(2.4 \times 10^{-9} \mu s / D a^{3}\right) \mathcal{M}^{3}$. Experimentally, we measure: $\tau_{\text {exp }} / c^{1.5}=((0.6 \pm 0.4) \times$ $\left.10^{-9} \mu \mathrm{s} / \mathrm{Da}^{3}\right) \mathcal{M}^{2.9 \pm 0.2}$ (Fig. 3). The prefactor is in good agreement with the expected value. There is good quantitative agreement between the experimental determination of the exponent of the power law dependency for large chains and the theoretical predictions of the reptation model [30], which confirms that under our experimental conditions entry is controlled by the phenomenon of chain extraction.

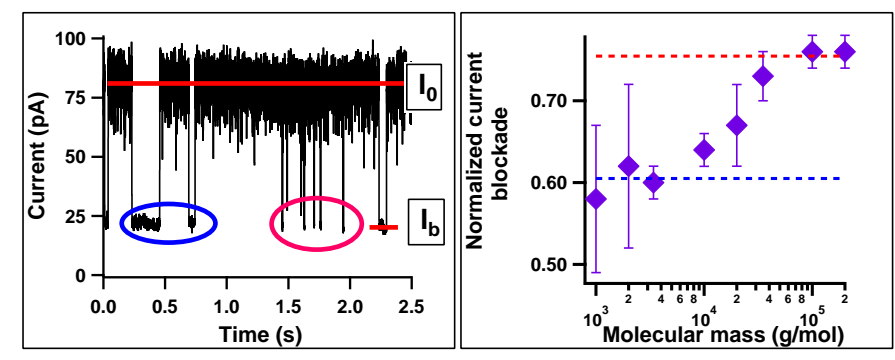

FIG. 4. a): Single-channel current trace through an $\alpha$ Hemolysin pore. We observe the dwell time of $200 \mathrm{kDa}$ (blue circle) $-35 \mathrm{kDa}$ (red circle) (2\%-98\%) mixture. b) Normalized current blockade $\left(1-\frac{I_{b}}{I_{0}}\right)$ as a function of molecular mass. The dotted lines correspond to the average values $0.61 \pm 0.02$ (blue) and $0.76 \pm 0.01$ (red).

Another observation of the transition between these two regimes is obtained by measuring the normalized current blockade (Fig. 4). For chains in the dilute regime, the amplitude of this blockade is $0.61 \pm 0.02$. This average value increases until $0.76 \pm 0.01$, when the chains are in the semi-dilute regime $(\mathcal{M}>35 k D a)$. In the first regime, the current blockade depends on the dwelling nature of each event (both bumping (with a low blockade) and translocations (with a large one)), as already observed [13]. In the second regime, we observe only translocations, which is why the current blockade is steady and does not vary with chain size.

Our experiments open the way to new studies, in semidilute solution, of the dynamics of macromolecules or biomolecules in confined geometry. The entry of small chains is possible when their radius of gyration is smaller than the pore diameter, in agreement with previous experiments, and obey the Daoud and de Gennes theories. The dynamics in the pore are governed by a diffusion motion due to the thermal energy. For the first time, the entry of large chains into the nanopore is observed, but only in the semi-dilute regime. The chain enters the pore as soon as the polymer reaches a threshold concentration corresponding to a mesh size with the same magnitude as the pore size, in agreement with the theories of de Gennes. We have demonstrated that the dynamics of long chains in the pore follow a reptation mechanism.

This work was supported by the Agence Nationale de 
la Recherche. We are grateful to Kari Foster for her attention to our manuscript and for kindly correcting the language of the manuscript.

* laurent.bacri@univ-evry.fr

[1] J. Zimmerberg and V. A. Parsegian, Nature 323, 36 (1986).

[2] I. Vodyanoy and S. M. Bezrukov, Biophys J 62, 10 (1992).

[3] S. M. Bezrukov and I. Vodyanoy, Biophys J 64, 16 (1993).

[4] S. M. Bezrukov, I. Vodyanoy, and V. A. Parsegian, Nature 370, 279 (1994).

[5] V. A. Parsegian, S. M. Bezrukov, and I. Vodyanoy, Biosci Rep 15, 503 (1995).

[6] S. Bezrukov, I. Vodyanoy, R. Brutyan, and J. Kasianowicz, Macromolecules 29, 8517 (1996).

[7] S. M. Bezrukov and J. J. Kasianowicz, Eur Biophys J 26, 471 (1997).

[8] L. Movileanu and H. Bayley, Proc Natl Acad Sci U S A 98, 10137 (2001).

[9] T. K. Rostovtseva, E. M. Nestorovich, and S. M. Bezrukov, Biophys J 82, 160 (2002).

[10] L. Movileanu, S. Cheley, and H. Bayley, Biophys J 85, 897 (2003).

[11] J. W. F. Robertson, C. G. Rodrigues, V. M. Stanford, K. A. Rubinson, O. V. Krasilnikov, and J. J. Kasianowicz, Proc Natl Acad Sci U S A 104, 8207 (2007).

[12] L. Bacri, A. Oukhaled, E. Hémon, F. B. Bassafoula, L. Auvray, and R. Daniel, Biochem Biophys Res Commun 412, 561 (2011).

[13] L. Brun, M. Pastoriza-Gallego, G. Oukhaled, J. Mathe, L. Bacri, L. Auvray, and J. Pelta, Phys Rev Lett 100, 158302 (2008).

[14] G. Gibrat, M. Pastoriza-Gallego, B. Thiebot, M.-F. Breton, L. Auvray, and J. Pelta, J Phys Chem B 112, 14687 (2008).

[15] R. J. Murphy and M. Muthukumar, J Chem Phys 126, 051101 (2007).

[16] Q. Chen, J. Liu, A. E. P. Schibel, H. S. White, and C. Wu, Macromolecules 43, 10594 (2010).
[17] G. Oukhaled, L. Bacri, J. Mathe, J. Pelta, and L. Auvray, Europhys Lett 82, 48003 (2008).

[18] M. Akeson, D. Branton, J. J. Kasianowicz, E. Brandin, and D. W. Deamer, Biophys J 77, 3227 (1999).

[19] M. Muthukumar, Annu Rev Biophys Biomol Struct 36, 435 (2007).

[20] J. J. Kasianowicz, E. Brandin, D. Branton, and D. W. Deamer, Proc Natl Acad Sci U S A 93, 13770 (1996).

[21] G. Oukhaled, J. Mathe, A.-L. Biance, L. Bacri, J.-M. Betton, D. Lairez, J. Pelta, and L. Auvray, Phys Rev Lett 98, 158101 (2007).

[22] R. Stefureac, L. Waldner, P. Howard, and J. Lee, Small 4, 59 (2008).

[23] M. Pastoriza-Gallego, L. Rabah, G. Gibrat, B. Thiebot, F. G. van der Goot, L. Auvray, J.-M. Betton, and J. Pelta, Journal of the American Chemical Society 133, 2923 (2011).

[24] B. Sakmann and E. Neher, Single-Channel Recording, 2nd ed. (Plenum Press, 1995).

[25] P. G. De Gennes, Scaling Concepts in Polymer Physics (Cornell University Press, 1979).

[26] M. Daoud and P. G. de Gennes, J. Phys. 38, 85 (1977).

[27] F. Brochard and P. G. De Gennes, J Chem Phys 67, 52 (1977).

[28] P.-G. de Gennes, Adv. Polym. Sci. 138, 91 (1999).

[29] J. E. Reiner, J. J. Kasianowicz, B. J. Nablo, and J. W. F. Robertson, Proc Natl Acad Sci U S A 107, 12080 (2010).

[30] F. Brochard and P. G. De Gennes, J. Phys. Lett. 40, L399 (1979).

[31] O. V. Krasilnikov and S. M. Bezrukov, Macromolecules 37, 2650 (2004).

[32] G. Guillot, L. Leger, and F. Rondelez, Macromolecules 18, 2531 (1985).

[33] J. Lal, Sunil K. Sinha, and Loïc Auvray, J. Phys. II France 7, 1597 (1997).

[34] E. P. Gilbert, L. Auvray, and J. Lal, Macromolecules 34, 4942 (2001).

[35] O. V. Krasilnikov, C. G. Rodrigues, and S. M. Bezrukov, Phys Rev Lett 97, 018301 (2006).

[36] P. Mueller, D. O. Rudin, H. Ti Tien, and W. C. Wescott, Nature 194, 979 (1962).

[37] M. Muthukumar, Phys Rev Lett 86, 3188 (2001). 